\title{
A chronic intracerebral cannula
}

\author{
MILAN REZEK and VIKTOR HAVLICEK \\ Department of Physiology, University of Manitoba, Winnipeg, R3E OW3, Canada
}

\begin{abstract}
The preparation of a chronic intracerebral cannula from disposable regular and dental needles is described. The cannula system has a convenient and reliable locking arrangement between the exteriorized part of the implanted cannula guide and a terminal plug of the extension tube. This arrangement enables to perform the infusions of experimental substances by a remote control in unrestrained, freely moving animals. Between infusions, the cannula guide is protected by a removable cap with fixed cleaning wire to prevent the infection and clogging.
\end{abstract}

The functional investigation of the central nervous system often involves repeated administration of experimental substances directly into the brain. In most instances, such procedures necessitate the use of intracerebral cannulas which are permanently implanted into the brain (i.e., Lilly, 1958). The delicate nature of intracerebral administrations requires the cannula systems which are accurate, reliable, and protected against the internal or external deteriorations caused either by clogging of the cannula guide or by behavior of the animal.

Present report describes the construction of an intracerebral cannula which may satisfy these requirements. The complete cannula system can be made from inexpensive parts which are either commonly available in laboratories or can be easily obtained from any laboratory supplier at low cost.

\section{PREPARATION OF THE CANNULA SYSTEM}

\section{Type 1}

Parts for the construction of this cannula system are: (a) two 23-ga. disposable needles (Monoject or Yale), (b) two 30-ga. dental disposable needles (Monoject 401 metal hub), (c) one 18-ga. disposable needle (Monoject or Yale), (d) two $1-\mathrm{ml}$ plastic disposable syringes, (e) polyethylene tubing fitting 30-ga. needle. All three basic components of this cannula system are assembled from these parts. These consist of: (a) intracerebral cannula guide and its exteriorized well, (b) a removable cap with a fixed cleaning wire and (c) a terminal arrangement of the extension tube which locks in to the well of the cannula guide. This latter component is interchangeable in this cannula system and need not be prepared separately for every cannula.

The cannula guide and its weil are prepared from a 23-ga. disposable needle as follows: the needle is divided into two sections, $1 \mathrm{~A}$ and $1 \mathrm{~B}$, by cutting it at the areas indicated by broken line in Figure 1. The lower section (1B) is further modified by filing its plastic part to a length of $4 \mathrm{~mm}$ when measured from the point where

This research was supported by the Medical Research Council of Canada and by the Sellers Foundation.
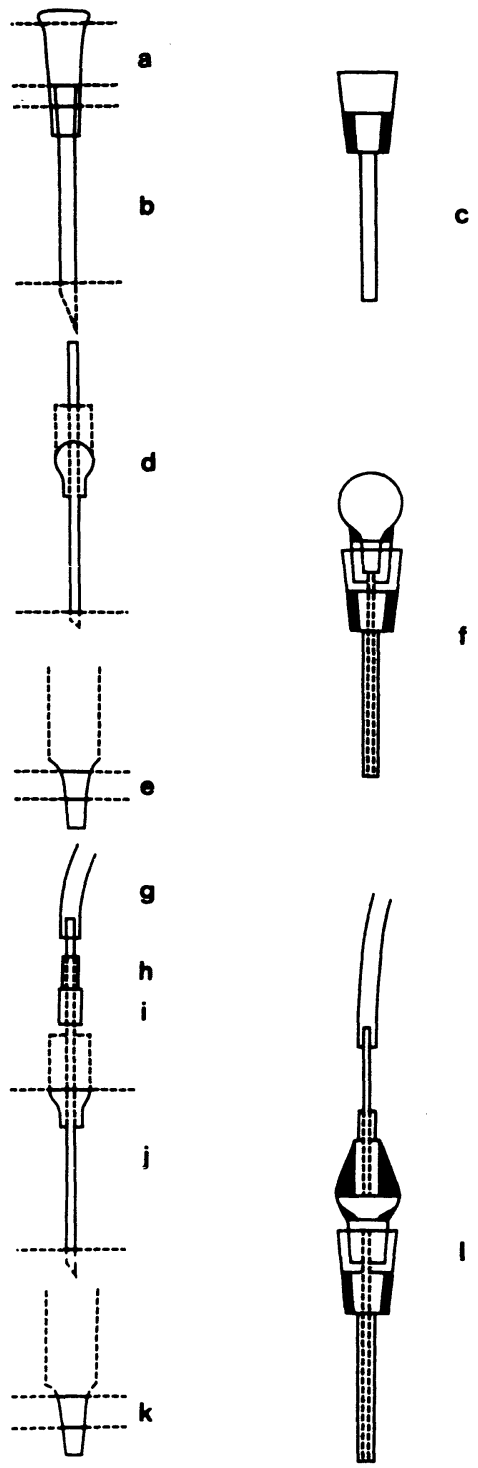

Figure 1. Components and assembly of the cannula system. 


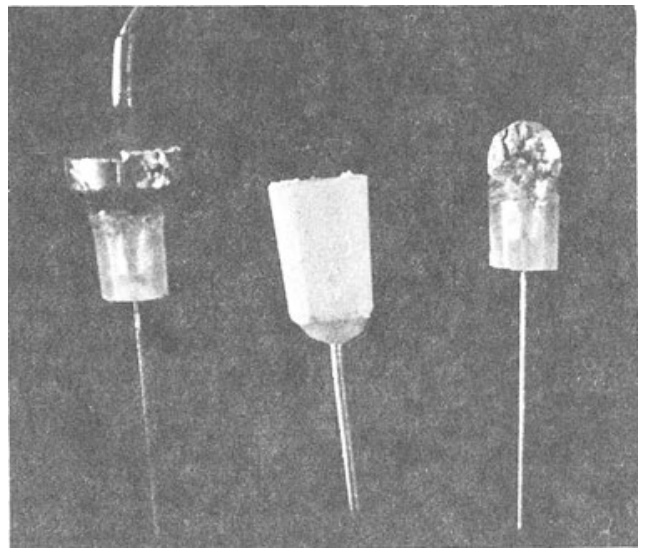

Figure 2. Completed components of the cannula system: terminal arrangement of the extension tube (left), cannula guide (middle), protective cap (right).

the metal shaft enters the plastic well and by slightly reducing its diameter while maintaining its conical shape. The diameter is reduced to the point where it can be tightly inserted into the upper part $(1 \mathrm{~A})$ as shown in Figure 1C. The two parts are then permanently connected by applying a small amount of epoxy from the bottom to the lower inner wall of Part $1 \mathrm{~A}$ while keeping Part 1B loosely inserted into Part 1A. Part 1B is then rotated to facilitate a proper adhesion of epoxy and to assure concentric position of $1 \mathrm{~B}$ within $1 \mathrm{~A}$. This procedure avoids the possibility of accidental application of epoxy on the upper inner wall of Part 1A where it would later interfere with a tight insertion of the removable cap or the plug of the extension tube in the well of the cannula guide.

The removable cap with its fixed cleaning wire is prepared from a 30-ga. disposable dental needle and a 1-ml plastic disposable syringe. First, the thin-walled aluminum well of the dental needle is completely flattened in a vise. Subsequently, the solid part of the needle, just below the well, which holds the 30-ga. tubing of the needle is gently rounded and reduced in diameter by filing so that it can be tightly inserted into part $1 \mathrm{E}$, made from the tip of the $1-\mathrm{ml}$ syringe. This connection is strengthened by applying the epoxy on the connecting surfaces of both parts before assembling them together. It is useful to insert the assembled cap into the cannula guide while epoxy is hardening to assure a proper concentric contact between both components. Finally, the flattened well of the needle is easily filed to a semicircular shape (Figure 1D and F) so that its edges do not exceed the diameter of Part $1 \mathrm{E}$.

The terminal assembly of the extension tube is prepared from a 30-ga. disposable dental needle, 1-ml plastic disposable syringe, and two supporting metal tubes made from 23-ga. and 18-ga. disposable needles. The construction of the lower part of the assembly which is inserted into the guiding cannula is very similar to the procedure for preparation of the removable cap. One difference is that the well of the needle is not flattened, but, instead, its upper part is cut off (Figure 1G). Subsequently parts of 23-ga. and 18-ga. needle tubing may be inserted concentrically on the upper part of $30 \mathrm{ga}$. dental needle as indicated by Figure $1 \mathrm{H}$ and $\mathrm{I}$ and fixed permanently by epoxy. Finally, the thin polyethylene tubing is inserted on the end of the 30-ga. needle. Before implanting the cannula, the needle of the extension tube is inserted into the guiding cannula and premeasured so that the tip of the inner 30-ga. needle exceeds the tip of cannula guide by $.5 \mathrm{~mm}$. The depth of insertion of Part $1 \mathrm{C}$ into the well of the cannula guide $(1 \mathrm{~A})$ should be marked on the edge of Part A while both components are still in the premeasured position. Both components are kept in this position also for implantation. The whole assembly is held in the stereotaxic apparatus by supporting tubes of the terminal plug of the extension tube. If necessary, a piece of 15-ga. needle tubing (about 1 in.) can be inserted tightly on the outer supporting tube (18-ga.) for a more convenient and stable fixation of the assembly in the stereotaxic apparatus.

The cannula system described can be used for both cerebral and intraventricular infusions. The exteriorized parts of these cannulas occupy a small space on the animal's head. This cannula system is presently used on rats and is easily anchored together with amphenol multielectrode connector on the animal's head. The advantages of presented cannula system include a practical combination of the external protective cap with a fixed cleaning wire and a convenient locking arrangement between the end of the extension tube and the cannula guide (Figure 2). This latter arrangement makes it possible to effect prolonged infusions safely in unrestrained animals. In our laboratory, animals are simultaneously connected to the infusion system and the recording EEG apparatus long before the infusion is started by a remote control from a separate observation and monitoring room. For prolonged infusions, it is useful to have a convenient counterweight arrangement or to have the polyethylene tubing of the extension tube protected by inserting it through a thin wire covering.

\section{REFERENCE}

Lilly, J. C. Electrode and cannulae implantation in the brain by a simple percutaneous method. Science, 1958, 127, 1181-1182.

(Received for publication December 20, 1974; revision accepted March $18,1975$. 